\title{
A Curvature Operator for a Regular Tetrahedron Shape in LQG*
}

\author{
O.Nemoul $^{\dagger}$ and N.Mebarki ${ }^{\ddagger}$ \\ Laboratoire de physique mathmatique et subatomique \\ Mentouri university, Constantine 1, Algeria
}

(Dated: March 7, 2018)

An alternative approach introducing a 3 dimensional Ricci scalar curvature quantum operator given in terms of volume and area as well as new edge length operators is proposed. An example of monochromatic 4-valent node intertwiner state (equilateral tetrahedra) is studied and the scalar curvature measure for a regular tetrahedron shape is constructed. It is shown that all regular tetrahedron states are in the negative scalar curvature regime and for the semi-classical limit the spectrum is very close to the Euclidean regime.

* This article is based on the work presented in the paper of ref. [28].

† omar.nemoul@yahoo.fr

$\ddagger$ nnmebarki@yahoo.fr 


\section{INTRODUCTION}

Loop Quantum Gravity (LQG) [1,2] is a background independent quantum field theory, it has been described as the best way to build a consistent quantum version of General Relativity (with vanishing cosmological constant $\Lambda=0$ ). Canonically, it is based on the implementation of the Holst action [3] and the Ashtekar-Barbero variables (The configuration variable is the real $s u(2)$ connection $A_{a}^{i}(x)$ and its canonical conjugate is the gravitational electric field $\left.E_{j}^{b}(x)\right)$ with a real Immirzi parameter $\gamma[4,5]$ by the Dirac quantization procedure [6]. In order to construct the starting kinematical Hilbert space, one has to use the well known representation of the holonomy-flux algebra [7]: it is represented by the space of all cylindrical wave functional through holonomies defined by the $s u(2)$ connection along a system of smooth oriented paths and flux variables as the smeared electric field along the dual surface for each path. Due to the background-independent property of LQG, it was possible to use Wilson loops [8] which are the natural gaugeinvariant holonomy of the gauge connection as a basis for the gauge invariant Hilbert space [9]. Another useful basis state of the quantum geometry known as the Penrose's spin networks is frequently used [10]. Spin network arises as a generalization of Wilson loops necessary to deal with mutually intersecting loops "nodes" which is represented by a space of intertwiners at each node [11]. One can construct well defined observables such as the area and volume acting on links and nodes respectively of smooth paths system [12]. The fuzziness and discreteness property of space [13-15] is predicted. A beautiful interpretation of the intertwiners in terms of quantum Euclidean polyhedra $[16,17]$ naturally arises. In this work, we construct a new geometrical information from LQG spin network based on the polyhedra interpretation of spin network states, which is the value of the 3d-Ricci scalar curvature and the edge length as a function of volume and boundary areas operators. A suggested introduction to the curvature operator in terms of the length operator and the dihedral angles was provided by using 3d- Regge calculus [18]. Moreover, there are three proposals for length operator discussed in refs. [19-21]. The main idea of our work comes from the determination of the volume and the boundary area of a fixed region in a Riemannian manifold as a function of the scalar curvature inside that region as well as its parameterization. One can invert these functions to get the explicit formula of the scalar curvature in terms of volume and boundary area of a fixed region. Similar idea can be done using a geodesic polyhedron shape ${ }^{1}$ [28]. By extending the Euclidean polyhedra interpretation to all non-zero curvature polyhedra, we can use the new proposed

${ }^{1}$ Geodesic polyhedron is the convex region enclosed by the intersection of geodesic surfaces. A geodesic surface is a surface with vanishing extrinsic curvature and the intersection of two such surfaces is necessarily a geodesic curve. 
scalar curvature operator related to a fixed polyhedron measure and try to determine its spectrum in order to know what kind of space in which the intertwiner state is represented. This geometrical approach can be considered as a natural arena for considering LQG including a cosmological constant. in the case of $\Lambda \neq 0$, The $S U(2)$ gauge invariant is still representing the kinematical space of LQG (since the cosmological constant just appears in the Hamiltonian constraint). Thus, one can describe the intertwiner state by a curved chunk of a curved polyhedron and then the main feature of our proposed curvature operator is to determine in a straightforward manner which cosmological constant value $\Lambda$ can an intertwiner state be interpreted as a fixed geodesic polyhedron. Moreover, a proposal to introduce a non-vanishing cosmological constant in LQG is to work with the q-deformed $\mathcal{U}_{q}(s u(2))$ rather than the $S U(2)$ itself [11, 22-25] and the use of curvature tetrahedron was suggested in [26]. In our approach, an example of a such monochromatic 4-valent node state was studied in details and its associated Kapovich-Millson phase space (i.e. the space of all equilateral Euclidean tetrahedron shapes) was constructed. Moreover, we will show the absence of a regular Euclidean tetrahedron from the volume orbit of relevant shapes in that phase space, instead of this it is possible to find a regular tetrahedron correspondence in the context of a non-zero constant curvature tetrahedron. It is worth to mention that the phase space of curved tetrahedron shapes idea has been initiated in ref. [27]. In our present paper, full expressions of volume and boundary face area of a regular tetrahedron in a constant curvature space (in terms of the scalar curvature and the edge length [28]) are explicitly derived than inverted to get the exact form of the 3d- Ricci scalar curvature and the edge length. At the quantum level, we obtain two well defined operators acting on the monochromatic 4 -valent nodes state. Their spectra show that all quantum atoms of space can be represented by chunks of regular hyperbolic tetrahedron of a negative curvature $R \sim-(8 \pi G h \gamma)^{-1}$. It also produces the Euclidean regular tetrahedron $R \sim 0$ in the semi-classical limit $j \gg 1$ ( $j$ is links color). In what follows, we will work in a unit where $8 \pi G h \gamma=1$. The paper is organized as follows : In section II, we give a motivation for a new scalar curvature measure. In section III, a strategy of defining new curvature operator in LQG is presented. In section IV, a 3d- Ricci scalar curvature and edge length operators are constructed for a regular tetrahedron state. Finally in section $\mathrm{V}$, we draw our conclusions.

\section{MOTIVATION FOR A NEW SCALAR CURVATURE MEASURE}

General relativity (GR) is a dynamical theory of spacetime within the framework of the general covariance. Accordingly, it should be described by geometrical observables with 
respect to other dynamical fields ${ }^{2}$. It implies that any invariant measure of GR can be written as a function of geometrical quantities. For instance, the 3d- Ricci scalar curvature in some point of the hypersurface $\Sigma_{t}$ embedded in a smooth Riemannian manifold $M$ is technically determined by the measure of volume and boundary area of a neighborhood region around this point. Doing it separately does not give enough geometrical informations of the space. Rather, it is mandatory to do this at the same time in order to get the complete information. To be more explicit, let us consider the simplest case of the 2 -sphere $S_{r(t)}^{2}$ of radius $r(t)$ in $2+1$ dimension (See Fig. 1). The spatial metric at a given time $t$ is:

$$
\left.d s^{2}\right|_{\Sigma_{t}}=r(t)^{2}\left(d \theta^{2}+\sin ^{2}(\theta) d \varphi^{2}\right)
$$

At $t=t_{0}$ we want to measure the $2 \mathrm{~d}$ - Ricci scalar curvature $R_{t_{0}}$ such that $r\left(t_{0}\right)=r_{0}$ This means we have to measure the radius $r_{0}$ (because $R_{t_{0}}=\frac{2}{r_{0}^{2}}$ ). To do so, we fix a region $\mathcal{D}_{a}^{S_{r_{0}}^{2}}(m)$ of a geodesic disc with a radius $a$ centering at a point $m \in S_{r_{0}}^{2}$ :

$$
\mathcal{D}_{a}^{S_{r_{0}}^{2}}(m)=\left\{p \in S_{r_{0}}^{2} \mid l_{m p}^{S_{r_{0}}^{2}} \leq a\right\} \subset S_{r_{0}}^{2}
$$

Where $l_{m p}^{S_{r_{0}}^{2}}$ is the geodesic length of the $S_{r_{0}}^{2}$ space between the points $m$ and $p$. The area $A\left(r_{0}, a\right)$ of the disc and its boundary curve length $L\left(r_{0}, a\right)$ are:

$$
\begin{gathered}
A\left(r_{0}, a\right)=2 \pi r_{0}^{2}\left(1-\cos \left(\frac{a}{r_{0}}\right)\right), \\
L\left(r_{0}, a\right)=2 \pi r_{0} \sin \left(\frac{a}{r_{0}}\right),
\end{gathered}
$$

Given the pair $\left(r_{0}, a\right)$, one can determine the area of a disc and its boundary curve length $(A, L)$. It is easy to invert these two functions to obtain:

$$
\begin{gathered}
R_{t_{0}}(A, L)=\frac{2}{r_{0}^{2}}=\frac{2\left(4 \pi A-L^{2}\right)}{A^{2}}, \\
a(A, L)=\frac{A}{\sqrt{4 \pi A-L^{2}}} \arctan \left(\frac{L \sqrt{4 \pi A-L^{2}}}{2 \pi A-L^{2}}\right),
\end{gathered}
$$

Thus, The simultaneous measurement of the area and the boundary curve length of a geodesic disc can allows us to estimate the value of the $2 \mathrm{~d}$ - Ricci scalar curvature $\left(R_{t_{0}}=\frac{2}{r_{0}^{2}}\right)$ and the disc radius $a$.

In $2+1$ dimension and for the 2 -sphere case, these two relations give us another way to measure the main important geometrical quantity which is the value of the $2 \mathrm{~d}$ - Ricci scalar curvature $R_{t_{0}}(A, L)$ as a function of the area measure and its boundary curve length

\footnotetext{
${ }^{2}$ Due to the diffeomorphism invariance property of GR. For details see [1].
} 
of a disc. Remarkably, this technique does not depend on the choice of the region; one can choose any shape of a region and get the same $2 \mathrm{~d}$ - scalar curvature. But how can we generalize this technique for arbitrary 3-dimensional topological spaces?. To get such a generalization, we try to find a relationship between the 3d- Ricci scalar curvature with the measurement of volume and boundary area of an arbitrary region. It was done by using small geodesic ball [29], and for any arbitrary regular tetrahedron in a constant curvature spaces [28]. The curvature can be determined by inverting the resulting functions in all cases.

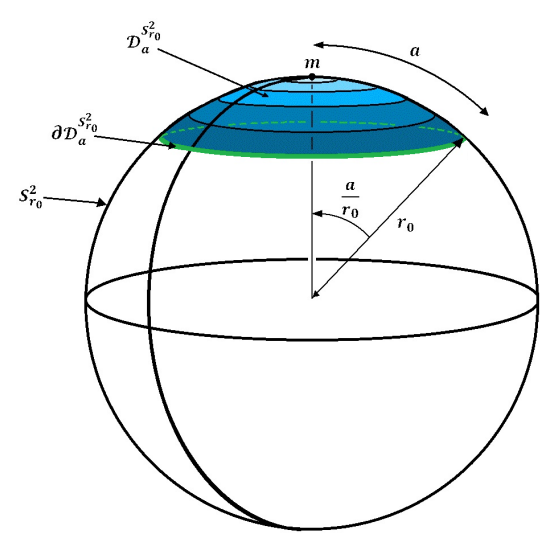

FIG. 1. The geodesic disc $\mathcal{D}_{a}^{S_{r_{0}}^{2}}$ (blue) and its boundary circle $\partial \mathcal{D}_{a}^{S_{r_{0}}^{2}}$ (green) in the 2-sphere $S_{r_{0}}^{2}$.

\section{STRATEGY FOR DEFINING A NEW CURVATURE OPERATOR IN LQG}

In loop quantum gravity $(\Lambda=0 \text { or } \Lambda \neq 0)^{3}$, the $S U(2)$ invariant Hilbert space at each F-valent node is the intertwiner space $\mathcal{H}_{F} \equiv i n v\left(V^{\left(j_{1}\right)} \otimes \cdots \otimes V^{\left(j_{F}\right)}\right)$ which is the quantization of the Kapovich-Millson phase space $\mathcal{S}_{F}$ i.e. the space of all Euclidean polyhedron shapes with fixed F-areas norms $\left\{A_{f} \sim j_{f}\right\}_{f=\overline{1, F}}$. This correspondence allows us to interpret each atom of space on a node (volume eigenstate) as quantum Euclidean polyhedra states. It offers infinite possible Euclidean polyhedra shapes for the same intertwiner state. In fact, after restricting the space of shapes of fixed areas $\left\{A_{f}\right\}_{f=\overline{1, F}}$ to a spectrum of volume operator, we will obtain $(2 F-5)$ dimensions hyper-surface of relevant shapes (since the $\mathcal{S}_{\left\{A_{f}\right\}}$ phase space has $2(F-3)$ dimensions). Now, it is legitimate to ask the following questions:

1. Is this correspondence unique?

2. Can Loop Quantum Gravity intertwiners states offer non-zero curvature grains of space?

${ }^{3}$ We consider the same $S U(2)$ gauge invariant kinematical Hilbert space for both $\Lambda=0$ and $\Lambda \neq 0$ cases. 
3. Can we find other polyhedra shapes possibilities in the non-zero curvature regime? For instance, the absence of the regular Euclidean tetrahedron correspondence with the monochromatic 4-valent node intertwiners, this means that there is no regular tetrahedron belonging to the volume orbits in the space of equilateral tetrahedra shapes; can we find this correspondence in the context of non-zero curvature spaces?

In what follows, we will focus on the two last questions by considering the generalization of this correspondence. In another words, we will interpret the intertwiner state by a fixed polyhedron shape (even if it doesn't belong to the volume orbit of Euclidean polyhedra shapes) and try to find out what kind of a curved space one must have in order that this polyhedron grain be nicely consistent with the area and volume spectra of LQG?. This means, we seek to find another possibilities of the correspondence in the context of other non-zero curvature polyhedra shapes. The task now is to determine new curvature operator related to a fixed polyhedron shape by using the approach similar to the one mentioned previously consisting in identifying the volume and areas operators of LQG with those of the corresponding polyhedron in an arbitrary curved space and inverting the resulting set of functions to end up to the classical and quantum formula of scalar curvature related to a fixed polyhedron. It is worth to mention that the classical consistency of the $3 \mathrm{~d}-$ Ricci scalar curvature measure as a function of the volume and boundary area measures is also well-defined at the quantum level since the commutativity between their associated geometrical operators ${ }^{4}$ is guaranteed in LQG. Unfortunately, we cannot exactly calculate the volume and boundary face area of a polyhedron in a general curved space, even if we make a perturbative series expansion around the Euclidean measure for a small polyhedron as it was mentioned for the small geodesic ball cases [29], we don't have any guidance to estimate the uncertainty of this expansion. The first problem occurred due the arbitrary degree of freedom of the considered general curved space. The solution is trivial; one can just relax the degree of freedom to spaces with a constant scalar curvature (one degree of freedom $)^{5}$. In fact, a spin network state of a fixed graph (dual to a fixed discretization) induces naturally a discrete locally valued function of the $3 \mathrm{~d}-$ Ricci scalar curvature. The reason is that all quantum geometric operators are not sensitive to all points inside the quantum atom of space; only nodes and links represent the quanta of space and its boundary surface respectively. Thus, each quantum atom of space corresponds to a constant $3 \mathrm{~d}-$ Ricci scalar curvature value, i.e. all points inside the quantum atom of space share the same geometrical property. In the following, we will make our calculation concerning the volume and boundary area of a polyhedron in a constant curvature Riemannian manifolds.

\footnotetext{
${ }^{4}$ In LQG, the volume and area operators are commute.

${ }^{5}$ The main reason is to describe LQG with a non-vanishing cosmological constant $\Lambda=$ cte.
} 
We remind that the Riemannian manifolds of a constant curvature can be classified into the Euclidean $\left(E u c^{3}, R=0\right)$, spherical $\left(S_{r}^{3}, R>0\right)$ and hyperbolic $\left(H_{r}^{3}, R<0\right)$ geometries (other spaces that have a constant curvature are isometric to the one of these three classes by the Killing-Hopf theorem $[30,31])$. As a byproduct, the full expression of volume and boundary face area of a regular tetrahedron in the 3-sphere $S_{r}^{3}$ and the 3-hyperbolic $H_{r}^{3}$ has been derived explicitly in terms of the $3 \mathrm{~d}$ - Ricci scalar curvature and the edge length in ref. [28]. In the monochromatic 4 -valent node example, we will be interested to study the possibility of finding a correspondence with a regular geodesic tetrahedron. Applying the 3d- Ricci scalar curvature operator related to a regular tetrahedron region on the intertwiner state for constructing a space of a constant curvature where one can have the regular tetrahedron correspondence for any irreducible representation $j$.

\section{APPLICATION: A MONOCHROMATIC 4-VALENT NODE STATE}

\section{IV.1. Quantum equilateral Euclidean tetrahedron}

The corresponding system of a monochromatic 4-valent intertwiner node is an equilateral Euclidean tetrahedron (tetrahedron with faces of equal areas, see Fig. 2 ) and the main ingredients that comprise this system can be summarized as follows:

\section{IV.1.1. Intertwiner space $\mathcal{H}_{4}$}

In LQG, the $S U(2)$ invariant Hilbert space of a monochromatic 4 -valent node $\left(j_{1}=\right.$ $\left.j_{2}=j_{3}=j_{4}=j\right)$ is the intertwiner space $\mathcal{H}_{4} \equiv i n v\left(V^{(j)} \otimes V^{(j)} \otimes V^{(j)} \otimes V^{(j)}\right)$ with a dimension $2 j+1$. There are two well-defined geometric operators acting on the gauge invariant intertwiner state $\left\{\left|\otimes_{l=1}^{4} j_{l}, i_{K}\right\rangle\right\}(l=\overline{1,4}, K=\overline{0,2 j})$ :

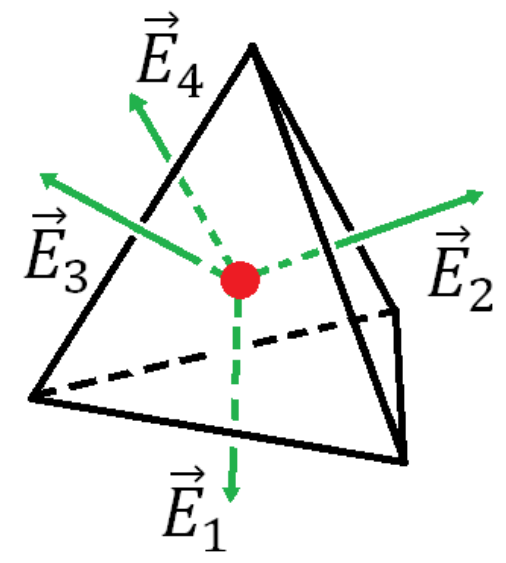

FIG. 2. Descriptions of the classical geometry of an equilateral Euclidean tetrahedron. 
The area operator acts trivially on the links as:

$$
\hat{A}_{f}\left|\otimes_{l=1}^{4} j_{l}, i_{K}\right\rangle=\sqrt{\left|\hat{\vec{E}}_{f}^{2}\right|}\left|\otimes_{l=1}^{4} j_{l}, i_{K}\right\rangle=\sqrt{j(j+1)}\left|\otimes_{l=1}^{4} j_{l}, i_{K}\right\rangle,
$$

The volume operator acts non-trivially on the node [17]:

$$
\hat{V}\left|\otimes_{l=1}^{4} j_{l}, i_{K}\right\rangle=\frac{\sqrt{2}}{3} \sqrt{\left|\hat{\vec{E}}_{1} \cdot\left(\hat{\vec{E}}_{2} \times \hat{\vec{E}}_{3}\right)\right|}\left|\otimes_{l=1}^{4} j_{l}, i_{K}\right\rangle \equiv \frac{\sqrt{2}}{3} \sqrt{\hat{Q}}\left|\otimes_{l=1}^{4} j_{l}, i_{K}\right\rangle,
$$

We have to diagonalize the volume matrix element by diagonalizing the matrix $\left[Q_{K^{\prime} K}^{(j)}\right]$ of elements:

$$
Q_{K^{\prime} K}^{(j)} \equiv\left\langle\otimes_{l=1}^{4} j_{l}, i_{K^{\prime}}|\hat{Q}| \otimes_{l=1}^{4} j_{l}, i_{K}\right\rangle
$$

with

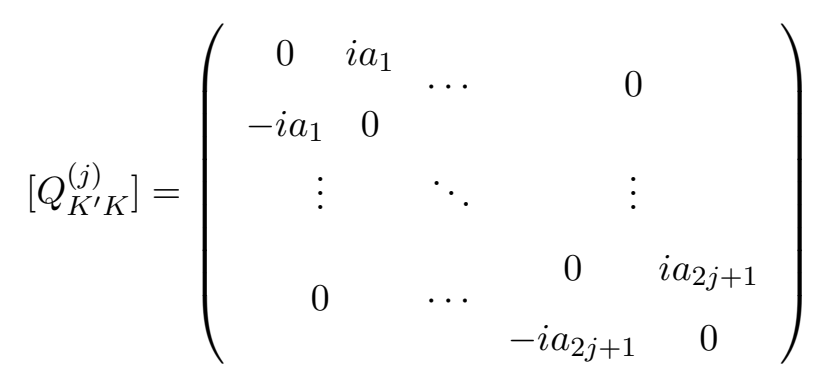

where

$$
a_{n}=\frac{1}{4} \frac{\left(n^{2}-(2 j+1)^{2}\right) n^{2}}{\sqrt{4 n^{2}-1}} \quad n=\overline{1,2 j+1}
$$

At each node, the quantum atoms of space is the common eigenstates ${ }^{6}\left\{\left|\otimes_{l=1}^{4} j_{l}, q_{K}\right\rangle\right\}$ of volume and area operators:

$$
\begin{gathered}
\hat{V}\left|\otimes_{l=1}^{4} j_{l}, q_{K}\right\rangle=V_{K}\left|\otimes_{l=1}^{4} j_{l}, q_{K}\right\rangle, \\
\hat{A}\left|\otimes_{l=1}^{4} j_{l}, q_{K}\right\rangle=\sqrt{j(j+1)}\left|\otimes_{l=1}^{4} j_{l}, q_{K}\right\rangle,
\end{gathered}
$$

\section{IV.1.2. The Kapovich-Millson phase space $\mathcal{S}_{4}$}

The space of all Euclidean equilateral tetrahedron [17] shapes with fixed areas norms $A_{1}=A_{2}=A_{3}=A_{4}=A=\sqrt{j(j+1)}$, satisfying the closure relation:

$$
\vec{A}_{1}+\vec{A}_{2}+\vec{A}_{3}+\vec{A}_{4}=\overrightarrow{0}
$$

The canonical coordinates are:

$$
p=\left|\vec{A}_{1}+\vec{A}_{2}\right| \quad q=\arccos \frac{\left(\vec{A}_{1} \times \vec{A}_{2}\right) \cdot\left(\vec{A}_{3} \times \vec{A}_{4}\right)}{\left|\vec{A}_{1} \times \vec{A}_{2}\right|\left|\vec{A}_{3} \times \vec{A}_{4}\right|}
$$

\footnotetext{
${ }^{6}$ They are vectors in the intertwiner space $\mathcal{H}_{4}:\left|\otimes_{l=1}^{4} j_{l}, q_{K}\right\rangle=\Sigma_{K^{\prime}=1}^{2 j+1} q_{K}^{K^{\prime}}\left|\otimes_{l=1}^{4} j_{l}, i_{K^{\prime}}\right\rangle$.
} 
It is obvious that

$$
0 \leq p \leq 2 A \quad-\frac{\pi}{2} \leq q \leq \frac{\pi}{2}
$$

All geometrical informations of an Euclidean equilateral tetrahedron can be constructed from its representation point $(p, q) \in \mathcal{S}_{4}$, such as the volume:

$$
V(A ; p, q)=\frac{\sqrt{2}}{3} \sqrt{\left|\vec{A}_{1} \cdot\left(\vec{A}_{2} \times \vec{A}_{3}\right)\right|}=\frac{1}{3 \sqrt{2}} \sqrt{|\sin (q)|\left(\frac{4 A^{2}}{p^{2}}-1\right)},
$$

Notice that the volume function has a maximal value as it is shown in Fig. 3. In fact, one has to solve the equations:

$$
\left.\frac{\partial V(A ; p, q)}{\partial p}\right|_{\left(p_{0}, q_{0}\right)}=\left.0 \quad \frac{\partial V(A ; p, q)}{\partial q}\right|_{\left(p_{0}, q_{0}\right)}=0
$$

It is easily to check that

$$
p_{0}=\frac{2 \sqrt{3}}{3} A \quad q_{0}= \pm \frac{\pi}{2}
$$

where

$$
V_{\text {max }}=V\left(A ; p_{0}, q_{0}\right)=2^{3 / 2} 3^{-7 / 4} A^{3 / 2},
$$

which is the expected Euclidean regular tetrahedron.

\section{IV.1.3. The correspondence $\mathcal{H}_{4} \leftrightarrow \mathcal{S}_{4}$}

Each volume spectrum (12) of the intertwiner space $\mathcal{H}_{4}$ corresponds to an orbit in the Kapovich-Millson phase space $\mathcal{S}_{4}$. These volume orbits are the possible Euclidean equilateral tetrahedron shapes of the volume eigenstate with a fixed face area norm $A=$ $\sqrt{j(j+1)}$ (See Fig. 4). 


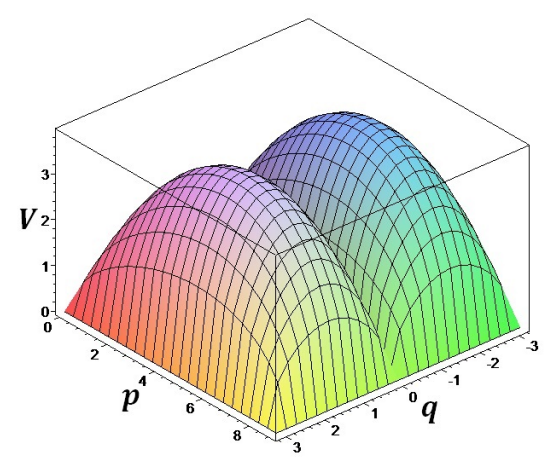

FIG. 3. The volume function in the the Kapovich-Millson phase space $\mathcal{S}_{4}$.

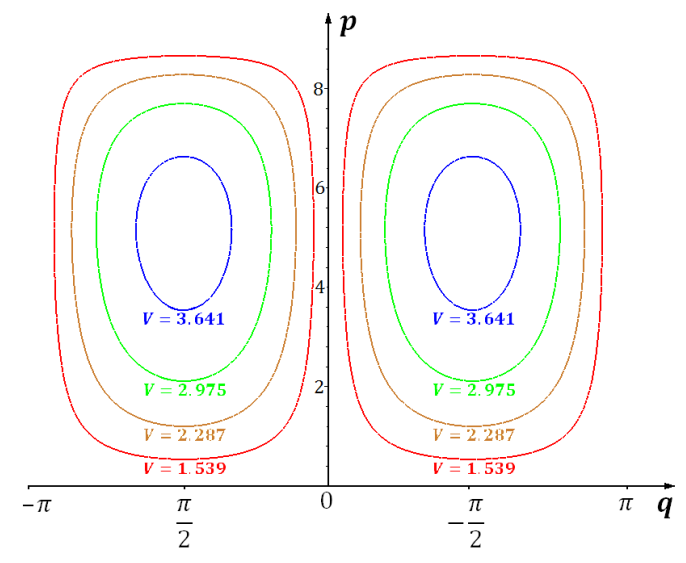

FIG. 4. The Kapovich-Millson phase space $\mathcal{S}_{4}$. The colored orbits are quantized levels of the volume operator in the monochromatic 4 -valent eigenstate of $j=4$.

The regular tetrahedron is the only state that has the maximum volume value. Therefore, the only atom of space state corresponds to a unique equilateral tetrahedron shape is the one that has a volume eigenvalue equal to the maximum volume of the phase space $\mathcal{S}_{F}$

$$
V_{\max }=2^{3 / 2} 3^{-7 / 4}(j(j+1))^{3 / 4},
$$

and it corresponds to the regular tetrahedron. In LQG, there is no quantum regular tetrahedron corresponding to a monochromatic 4-valent node state, since all quantum volume spectra are below the volume of a regular tetrahedron with a face area $A=\sqrt{j(j+1)}$ (See Fig. 5). The existence of a such regular tetrahedron solution is guaranteed by the correspondence of the 4 -valent node intertwiner space $\mathcal{H}_{4}$ with a new generalized KapovichMillson phase space $\mathcal{S}_{\{\mathbf{4}, \boldsymbol{R}\}}$ of equilateral tetrahedra shapes in constant curvature space $R[27]$

$$
\mathcal{H}_{4} \leftrightarrow \mathcal{S}_{\{4, R\}},
$$




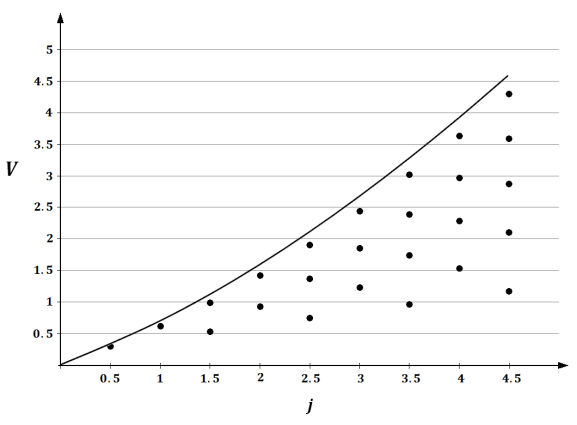

FIG. 5. Comparison of the regular Euclidean tetrahedron volume (dark line) with the LQG volume spectra (dots) for the monochromatic 4 -valent node state with different links color $j$.

IV.2. Ricci scalar curvature and edge length operators for regular tetrahedron state

Now, let us look for the 3d- Ricci scalar curvature value in which one can represent the monochromatic 4-valent quanta of space as a regular tetrahedron in a constant curvature space, in other words we seek to study the possibility of the regular tetrahedron state existence in a new equivalent Kapovich-Millson phase space $\mathcal{S}_{\{\mathbf{4}, \boldsymbol{R}\}}$ in the context of constant curvature spaces. In reference [28], the volume and the boundary face area of a regular spherical and hyperbolic tetrahedron given as explicit functions of the edge length $a$ and the radius $r=\sqrt{\frac{6}{|R|}}$ are shown to have the following expressions: ${ }^{7}$

$$
\begin{aligned}
& A^{\Sigma}(r, a)=\epsilon^{2} r^{2}\left(3 \arccos \left(\frac{\cos \left(\frac{a}{\epsilon r}\right)}{\cos \left(\frac{a}{\epsilon r}\right)+1}\right)-\pi\right), \\
& V^{\Sigma}(r, a)=12 \epsilon^{3} r^{3} \int_{0}^{\tan \left(\frac{a}{2 \epsilon r}\right)} d t \frac{t \arctan (t)}{\left(3-t^{2}\right) \sqrt{2-t^{2}}},
\end{aligned}
$$

where

$$
\epsilon=\left\{\begin{array}{cc}
1 & \Sigma=S_{r}^{3} \\
i & \Sigma=H_{r}^{3}
\end{array},\right.
$$

The Euclidean case is well-defined in the limit $r \rightarrow \infty$. A direct application of the resulted formulas $(23,24)$ in LQG is to find a $3 \mathrm{~d}$ - scalar curvature of the quantum atom of space such that the monochromatic 4-valent node has an interpretation of a regular tetrahedron in a constant curvature space. For each area and volume spectra of the operators $(12,13)$, inverting analytically these systems of functions is not so simple instead, we can deal with it numerically and construct the 3d- Ricci scalar curvature and the edge length spectra

\footnotetext{
${ }^{7}$ Notice that the geodesic surfaces of the $S_{r}^{3}$ and $H_{r}^{3}$ are portions of the great 2-dimensional spheres $S_{r}^{2}$ and hyperbolic $H_{r}^{2}$ respectively. Indeed, the area expression (24) of a regular triangle is a combination of the area formula given by the dihedral angle $\Theta$ and the $\operatorname{cosine}$ rule $\cos (\Theta)=\frac{\cos \left(\frac{a}{\epsilon r}\right)}{\cos \left(\frac{a}{\epsilon r}\right)+1}$ in the context of spherical and hyperbolic trigonometry.
} 
(See Figs. 6). In Figs. 6a, 6b and 6c, each curve with the same color corresponds to volume, scalar curvature and edge length spectra of the same states

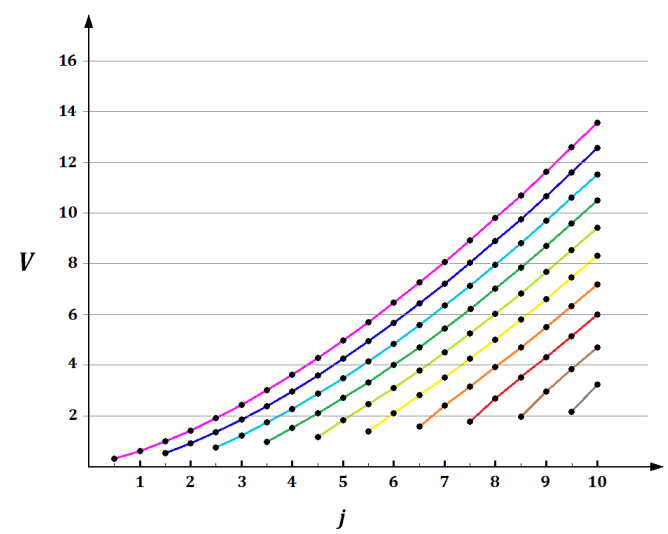

$(6 a)$

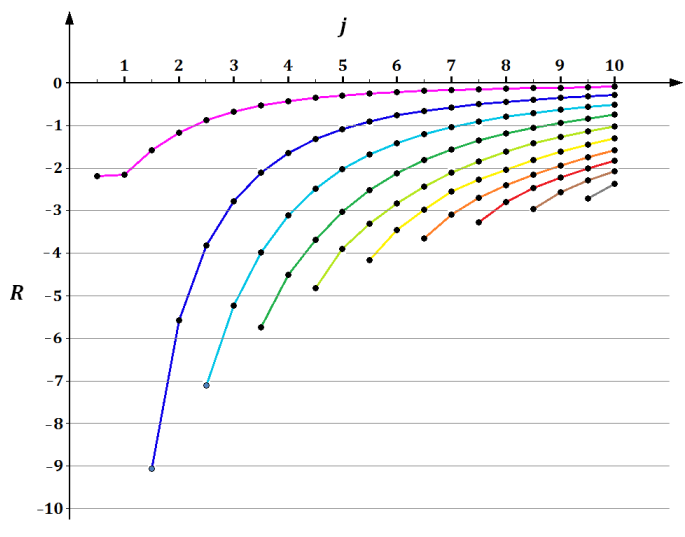

$(6 b)$

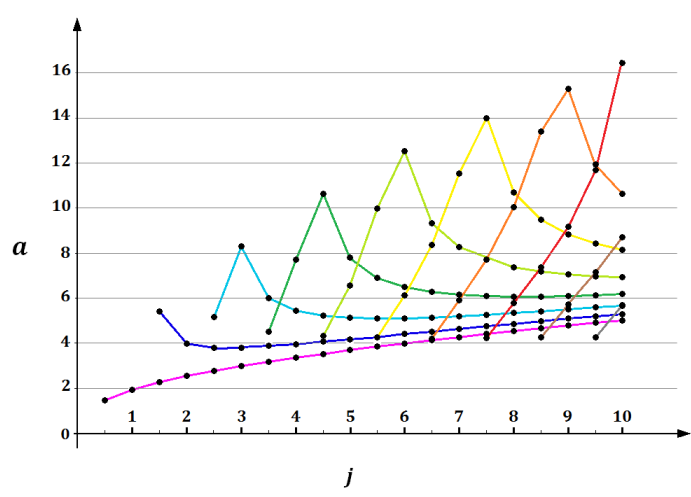

$(6 c)$

FIG. 6. Colored lines of different spectra levels for volume (6a), scalar curvature (6b) and edge length (6c) of a monochromatic 4-valent intertwiner.

From the the above figures 6 , it is worth to shed light on the main following conclusions:

1. The existence of a regular tetrahedron consistent with LQG data (volume and area spectra) is guaranteed in the negative curvature regime, and then one can represent the monochromatic 4 -valent state by a regular hyperbolic tetrahedron.

2. In general speaking, the 4-valent monochromatic state that has a biggest volume represented by a regular tetrahedron in negative constant curvature space is the closest to the Euclidean space with the smallest edge length and vice versa.

3. The lowest level value of the edges length (violet curve in Fig. 6c) are approximately the edges length of the Euclidean regular tetrahedron with a face area 
$A=\sqrt{j(j+1)}$

$$
a_{\min } \approx\left(\frac{4 A}{\sqrt{3}}\right)^{\frac{1}{2}}=\left(\frac{4 \sqrt{j(j+1)}}{\sqrt{3}}\right)^{\frac{1}{2}}
$$

4. For a generic spin value $j \sim 1$, we find that the regular tetrahedron solutions of negative scalar curvature spectra are in the range:

$$
R \sim-(8 \pi G h \gamma)^{-1} \sim-10^{70} / \gamma m^{-2}
$$

5. In the semi-classical limit $j \gg 1$, the monochromatic 4 -valent will be more closer to be identified with the Euclidean regular tetrahedron, because all scalar curvature spectra vanish as well as the edge length spectra tend asymptotically to the edge length of a regular Euclidean tetrahedron given in (26) (See Figs. 6b, 6c). Accordingly, we are able to have a good approximation of the volume and boundary face area functions $(23,24)$ around the zero constant curvature in the case of $j \gg 1$. In fact, by expanding these two functions $(23,24)$ with respect to the variable $\frac{a}{r}$, we obtain:

$$
\begin{gathered}
A^{\Sigma}(r, a)=\frac{\sqrt{3}}{4} a^{2}\left[1+\frac{1}{8}\left(\frac{a}{\epsilon r}\right)^{2}+\mathcal{O}\left(\left(\frac{a}{\epsilon r}\right)^{4}\right)\right], \\
V^{\Sigma}(r, a)=\frac{\sqrt{2}}{12} a^{3}\left[1+\frac{23}{80}\left(\frac{a}{\epsilon r}\right)^{2}+\mathcal{O}\left(\left(\frac{a}{\epsilon r}\right)^{4}\right)\right],
\end{gathered}
$$

As we have previously said, the analytic inversion of the two functions $(23,24)$ is not analytically possible, instead of doing the exact inversion with respect to the exact variables $(r, a)$, we will use the good approximation functions $(28,29)$ with respect to the approximate variables $(\tilde{r}, \tilde{a})$ and write:

$$
\begin{gathered}
A^{\Sigma}(\tilde{r}, \tilde{a})=\frac{\sqrt{3}}{4} \tilde{a}^{2}\left(1+\frac{1}{8} \tilde{x}\right), \\
V^{\Sigma}(\tilde{r}, \tilde{a})=\frac{\sqrt{2}}{12} \tilde{a}^{3}\left(1+\frac{23}{80} \tilde{x}\right),
\end{gathered}
$$

where

$$
\tilde{x}=\left(\frac{\tilde{a}}{\epsilon \tilde{r}}\right)^{2}=\frac{\tilde{R} \tilde{a}^{2}}{6}
$$

Inverting the two functions $(30,31)$ for the two variables $\tilde{R}$ and $\tilde{a}$, we obtain approximated formulas of the scalar curvature as well as the edge length:

$$
\tilde{R}(A, V)=3 \frac{\sqrt{3}}{2 A} \tilde{x}\left(1+\frac{1}{8} \tilde{x}\right),
$$




$$
\tilde{a}(A, V)=\left(\frac{4 \sqrt{3}}{3} \frac{A}{1+\frac{1}{8} \tilde{x}}\right)^{1 / 2}
$$

where

$$
\begin{gathered}
\tilde{x}(A, V)=\frac{4 \sqrt{3} A}{F(A, V)}-8, \\
F(A, V)=\left[\frac{1}{78} G(A, V)+\frac{23 \sqrt{3} A}{G(A, V)}\right]^{2},
\end{gathered}
$$

and

$$
G(A, V)=\left(-205335 \sqrt{3} V+117 \sqrt{-1265368 \sqrt{3} A^{3}+9240075 V^{2}}\right)^{1 / 3},
$$

Now, one has to quantize the 3d- Ricci scalar curvature and edge length functions given in $(33,34)$ by quantizing the area and volume operators to obtain quantum operators that act on the state of monochromatic 4-valent node quantum atom of space (the volume eigenstate):

$$
\begin{gathered}
\tilde{R}(A, V) \rightarrow \hat{\tilde{R}}(\hat{A}, \hat{V}), \\
\tilde{a}(A, V) \rightarrow \hat{\tilde{a}}(\hat{A}, \hat{V}),
\end{gathered}
$$

As the color $j$ increases, the accuracy of these two operators $(38,39)$ will be very high and their behavior spectra for $j \rightarrow \infty$ in the semi-classical limit is well known and it gives the Euclidean solution (See table I)

$$
\begin{gathered}
\hat{\tilde{R}}(\hat{A}, \hat{V})\left|\otimes_{\substack{l=1 \\
j \rightarrow \infty}}^{4} j_{l}, q_{K}\right\rangle=\tilde{R}_{K}\left(\sqrt{j(j+1)}, V_{K}\right)\left|\otimes_{\substack{l=1 \\
j \rightarrow \infty}}^{4} j_{l}, q_{K}\right\rangle \approx 0, \\
\hat{\tilde{a}}(\hat{A}, \hat{V})\left|\otimes_{\substack{l=1 \\
j \rightarrow \infty}}^{4} j_{l}, q_{K}\right\rangle=\tilde{a}_{K}\left(\sqrt{j(j+1)}, V_{K}\right) \underset{\substack{l=1 \\
j \rightarrow \infty}}{\left|\otimes_{l}, q_{K}\right\rangle}=\left(\frac{4 j}{\sqrt{3}}\right)^{\frac{1}{2}}\left|\otimes_{\substack{l=1 \\
j \rightarrow \infty}}^{4} j_{l}, q_{K}\right\rangle,
\end{gathered}
$$

\section{CONCLUSION}

We have found a new approach of measuring the 3d- Ricci scalar curvature value by measuring the volume of a region and its boundary area. We have applied this technique in LQG by generalizing the interpretation of the intertwiner state to all constant curvature spaces. In the context of a non-vanishing cosmological constant, the main feature of our proposed curvature operator is to determine in a straightforward manner which cosmological constant value can an intertwiner state be interpreted as a geodesic polyhedron. As a byproduct, we have studied the possibility of finding the regular tetrahedron correspondence with the monochromatic 4 -valent node in other constant curvature spaces. It 
is shown that all regular tetrahedron states are in the negative scalar curvature regime; for $j \gg 1$ the scalar curvature spectrum will be very close to the Euclidean regime. We conclude that the simultaneous measure ${ }^{8}$ of the volume and the boundary area of the monochromatic 4-valent node state allow us to estimate the appropriate case of a constant curvature space in which this state can be interpreted as a regular tetrahedron.

TABLE I. Comparison of the approximated spectra of the two operators $(\hat{\widetilde{R}}, \hat{\tilde{a}})$ associated to a regular tetrahedron with their exact value $(R, a)$ for the highest volume level (violet curve in Fig. 6 a) of the monochromatic 4 -valent node state for $j=1,2,3, \ldots, 10$.

\begin{tabular}{ccccccccc}
\hline \hline$j$ & $A$ & $V_{\max }$ & $R$ & $\widetilde{R}$ & $\delta R \%$ & $a$ & $\widetilde{a}$ & $\delta a \%$ \\
\hline 1 & 1.414 & 0.620 & -2.146 & -1.418 & $34 \%$ & 1.954 & 1.914 & $2.07 \%$ \\
2 & 2.449 & 1.425 & -1.156 & -0.782 & $32 \%$ & 2.557 & 2.511 & $1.82 \%$ \\
3 & 3.464 & 2.444 & -0.663 & -0.478 & $28 \%$ & 2.998 & 2.960 & $1.25 \%$ \\
4 & 4.472 & 3.641 & $-0,422$ & -0.320 & $24 \%$ & 3.369 & 3.340 & $0.87 \%$ \\
5 & 5.477 & 4.990 & -0.291 & -0.229 & $21 \%$ & 3.700 & 3.677 & $0.63 \%$ \\
6 & 6.481 & 6.476 & -0.212 & -0.172 & $19 \%$ & 4.003 & 3.983 & $0.48 \%$ \\
7 & 7.483 & 8.086 & -0.161 & -0.134 & $17 \%$ & 4.283 & 4.267 & $0.37 \%$ \\
8 & 8.485 & 9.812 & -0.127 & -0.107 & $15 \%$ & 4.545 & 4.532 & $0.30 \%$ \\
9 & 9.487 & 11.646 & -0.102 & -0.088 & $14 \%$ & 4.793 & 4.782 & $0.24 \%$ \\
10 & 10.488 & 13.583 & -0.084 & -0.073 & $13 \%$ & 5.029 & 5.019 & $0.20 \%$ \\
\hline \hline
\end{tabular}

[1] C. Rovelli, Quantum gravity, Cambridge university press (2004), cpt.univ-mrs.

[2] T. Thiemann, Introduction to modern canonical quantum general relativity, Cambridge University Press (2007), arXiv:gr-qc/0110034.

[3] Holst, Sren, Barbero's Hamiltonian derived from a generalized Hilbert-Palatini action, Phys. Rev. D. 53 (10): 59665969 (1996), arXiv:gr-qc/9511026.

[4] A. Ashtekar, New variables for classical and quantum Gravity, Phys. Rev. Lett. 57, 2244-2247 (1986).

[5] J. F. Barbero, Real Ashtekar variables for Lorentzian signature space times, Phys. Rev. D 51, 5507-5510 (1995), arXiv:gr-qc/9410014.

[6] P. Dirac, Lectures on Quantum Mechanics, (Belfer Graduate School of Science, Yeshiva University Press,New York 1964).

[7] J. Lewandowski, A. Okolow, H. Sahlmann, T. Thiemann, Uniqueness of diffeomorphism invariant states on holonomyflux algebras, Commun. Math. Phys. 267: 703-733, (2006), arXiv:grqc/0504147.

[8] Wilson. K, Confinement of quarks, Phys. Rev. D. 10 (8): 2445 (1974).

\footnotetext{
${ }^{8}$ At the quantum level, the commutativity is well-defined between volume and area operators.
} 
[9] C. Rovelli, L. Smolin, Knot Theory and Quantum Gravity, Phys. Rev. Lett, 61 (10): 11551958 (1988).

[10] R. Penrose (1971a), Angular momentum: an approach to combinatorial spacetime, in T. Bastin (ed.). R. Penrose (1971b), Applications of negative dimensional tensors, in D. J. A. Welsh (ed.).

[11] C. Rovelli, L. Smolin, Spin networks and quantum gravity, Phys. Rev. D. 52 (10): 57435759 (1995), arXiv:gr-qc/9505006.

[12] P. Don, S. Speziale, Introductory lectures to loop quantum gravity, lectures given at the 3eme Ecole de Physique Theorique de Jijel, Algeria (2009), arXiv:gr-qc/1007.0402v2.

[13] C. Rovelli, L. Smolin, Discreteness of area and volume in quantum gravity, Nucl. Phys. B 442: 593-622 (1995), arXiv:gr-qc/9411005.

[14] A. Ashtekar, J. Lewandowski. Quantum theory of geometry. I: Area operators, Class. Quant. Grav. 14: A55-A82 (1997), arXiv:gr-qc/9602046.

[15] A. Ashtekar, J. Lewandowski. Quantum theory of geometry. II: Volume operators. Adv. Theor. Math. Phys. 1: 388-429 (1998), arXiv:gr-qc/9711031.

[16] E. Bianchi, P. Dona and S. Speziale, Polyhedra in loop quantum gravity, Phys. Rev. D 83, 044035 (2011), arXiv:gr-qc/1009.3402v2.

[17] E. Bianchi, Hal M. Haggard, Bohr-sommerfeld quantization of space, Phys. Rev. D, 86(12): 124010 (2012), arXiv:gr-qc/1208.2228.

[18] E. Alesci, M. Assanioussi, J. Lewandowski, A curvature operator for LQG, Phys. Rev. D 89, 124017 (2014), arXiv:gr-qc/1403.3190v4.

[19] T. Thiemann, A Length operator for canonical quantum gravity, J. Math. Phys. 39, 3372 (1998), arXiv:gr-qc/9606092.

[20] E. Bianchi, The Length operator in Loop Quantum Gravity, Nucl. Phys. B 807, 591 (2009), arXiv:gr-qc/0806.4710.

[21] Y. Ma, C. Soo, J. Yang, New length operator for loop quantum gravity, Phys. Rev. D 81, 124026 (2010), arXiv:gr-qc/1004.1063.

[22] V. G. Turaev, O. Y. Viro, State sum invariants of 3 manifolds and quantum $6 \mathrm{j}$ symbols," Topology 31865 (1992).

[23] M. Dupuis, F. Girelli, Observables in Loop Quantum Gravity with a cosmological constant, Phys. Rev. D 90, 104037 (2014), arXiv:gr-qc/1311.6841.

[24] Ma. Dupuis, F. Girelli, Quantum hyperbolic geometry in loop quantum gravity with cosmological constant, Phys. Rev. D87: 121502 (2013), arXiv:gr-qc/1307.5461.

[25] V. Bonzom, M. Dupuis, F. Girelli, E. R. Livine, Deformed phase space for 3d loop gravity and hyperbolic discrete geometries, (2014), arXiv:gr-qc/1402.2323.

[26] Y. Taylor and C. Woodward, 6j symbols for Uq (sl2) and non-Euclidean tetrahedra, Sel. Math. New. Ser. 11, 539 (2005), arXiv:math.QA/0305113.

[27] H. M. Haggard, Mu. Han, A. Riello, Encoding Curved Tetrahedra in Face Holonomies: a Phase Space of Shapes from Group-Valued Moment Maps, Annales Henri Poincaré 17 no.8, 2001-2048 (2016), arXiv:gr-qc/1506.03053. 
[28] O. Nemoul, N. Mebarki, Volume and Boundary Face Area of a Regular Tetrahedron in a Constant Curvature Space,(2018), arXiv:gen-ph/1803.10809.

[29] A. Gray, The volume of a small geodesic ball of a Riemannian manifold, Michigan Math. J. 20 (1974).

[30] H. Hopf, Zum Clifford-Kleinschen Raumproblem, Mathematische Annalen, Springer Berlin Heidelberg, 95: 313339 (1926).

[31] W. Killing, Ueber die Clifford-Klein'schen Raumformen, Mathematische Annalen, Springer Berlin Heidelberg, 39: 257278 (1891). 